SEMBLANZAS 



\title{
GUMERSINDO DE AZCÁRATE: DERECHO, SELFGOVERNMENT Y CONSTITUCIÓN INGLESA
}

\author{
GONZALO CAPELLÁN DE MIGUEL \\ Profesor Titular de Historia Contemporánea \\ Universidad de La Rioja
}

\begin{abstract}
SUMARIO
I. El maestro y su influjo sociopolítico desde la cátedra. II. Azcárate y la generación de 1868: la constitución de 1869 como referente de libertades, derecho y democracia. III. La Restauración: el selfgovernment y el modelo constitucional de Inglaterra
\end{abstract}

\section{EL MAESTRO Y SU INFLUJO SOCIOPOLÍTICO DESDE LA CÁTEDRA}

«Maestro de maestros». Con estas significativas palabras se refirió a Gumersindo de Azcárate y Méndez (León, 1840 - Madrid, 1917) el Decano de la Facultad de Derecho de la Universidad de Madrid, Rafael de Ureña, en 1918. Lo hacía en las páginas destinadas a presentar una publicación clave en el ámbito académico del derecho español del siglo xx, Revista de Ciencias Jurídicas y Sociales, que la propia Facultad de Derecho instituía en ese momento como órgano de expresión ${ }^{1}$.

El hecho, en absoluto casual, de que ese primer número de la revista comenzara con un artículo de Rafael Altamira dedicado a Gumersindo de Azcárate, recién fallecido, nos habla por sí mismo de la relevancia del jurista y político leonés. En dicho artículo Altamira destaca dos facetas claves a tener en cuenta a la hora de trazar una semblanza de Azcárate. En primer lugar, refuerza la idea enfatizada por Ureña sobre la consideración de Azcárate como «el maestro»². Un

1 R. DE UreñA, «Nuestros propósitos», Revista de Ciencias Jurídicas y Sociales, Año I, n. ${ }^{\circ}$ 1, 1918, p. 6.

2 Su trabajo para obtener el grado de doctor, centrado en la Historia de la propiedad comunal, se realizó bajo la dirección de Azcárate. Altamira, que logró la Catedra de Historia del Derecho de la Universidad de Oviedo en 1897 y fue uno de los miembros fundadores de la Facultad de Derecho de esta Universidad, 
reconocimiento que destaca su dimensión académica, como «uno de los hombres de más bienhechora y positiva influencia en la vida universitaria española» y «el tipo modelo del universitario» ${ }^{3}$. Una valoración ganada a lo largo del casi medio siglo que transcurrió entre su entrada en la Universidad Central de Madrid como Profesor auxiliar de la Facultad de Derecho en el curso 1868/69 para explicar Economía política, su actividad como catedrático numerario en 1873 , enseñando la asignatura de Legislación comparada en la misma Facultad y su jubilación en septiembre de1915. Pocos meses después, el rey Alfonso XIII, a propuesta del Consejo de Ministros, nombraba a Azcárate «Rector honorario» de la Universidad Central, un galardón que nadie hasta entonces había alcanzado.

En la exposición de motivos previa al Real Decreto, el Ministerio de Instrucción Pública y Bellas Artes le definía de nuevo como «el maestro» y «el hombre del apostolado del derecho», que había ejercido de generación en generación, trasmitiendo los «tesoros de su ciencia» de unos a otros discípulos. Una labor que, se podía leer en la Gaceta de Madrid, «es en la vida universitaria algo así como la plenitud del Magisterio, en trascendencia educadora y en influjo social, del mismo modo que en la cultura de España es una claridad orientadora y en el Parlamento una magistratura de excepción» ${ }^{4}$.

Y esas palabras conducen directamente hasta el segundo aspecto a destacar en el artículo de Altamira: la implicación de Azcárate en la vida de la España de su época. El ilustre profesor nunca constriñó su labor académica a los muros de la universidad, sino que trascendió su ejercicio docente desde la cátedra en numerosos momentos y ámbitos, como tendremos ocasión de ver a lo largo de esta semblanza. Eso es a lo que, con toda probabilidad, se refería Altamira al afirmar que Azcárate «lleva lo propio de su función docente a todos los ámbitos de la vida nacional. Con él pudo decirse, mejor que en otros casos de la historia contemporánea, que la Universidad gobernó el país entero, sin perder ninguna de sus nobles cualidades» ${ }^{5}$.

Esa actuación bifronte en el ámbito científico-académico como profesor de derecho y escritor, al tiempo que una consagración personal a diferentes asociaciones, academias e instituciones diversas en el mundo cultural, político y social fueron características de la polifacética vida de Azcárate. Pero se podría decir que en todas ellas el nexo común fue la extraordinaria importancia concedida al derecho, entendido no solo como un fin fundamental de la vida cuya institución propia era el Estado, sino también como la relación que — sin suplantarlas- el derecho debía tener con las restantes esferas de la vida del hombre (económica,

realizó una remarcable contribución académica, entre otros campos, a la historia del derecho y al derecho internacional. Vid. E. M. ${ }^{a}$ Valero Juan y E. Rubio Cremades, Rafael Altamira: historia, literatura y derecho, Alicante, Universidad de Alicante, 2004.

3 R. Altamira, «Azcárate», Revista de Ciencias Jurídicas y Sociales, Año I, n. ${ }^{o}$ 1, 1918, pp. 7-8.

4 Gaceta de Madrid, n. ${ }^{\circ}$ 3, 5 de enero de 1916, p. 14.

5 R. Altamira, «Azcárate», art. cit., p.8. 
científica, social...) en tanto que presta las condiciones para el adecuado cumplimiento del resto de los fines humanos.

\section{AZCÁRATE Y LA GENERACIÓN DE 1868: LA CONSTITUCIÓN DE 1869 COMO REFERENTE DE LIBERTADES, DERECHO Y DEMOCRACIA}

En el citado número inaugural de la Revista de Ciencias Jurídicas y Sociales se decidió acompañar el artículo de homenaje a Azcárate con el texto de su discurso para la obtención del doctorado en Derecho Civil y Canónico en junio de 1869, un juicio crítico sobre la Ley 61 de Toro. Si ya hemos visto que el fin de su carrera docente y de su vida se cerraron con un reconocimiento que deja fuera de cuestión su relevancia en el mundo académico del derecho español contemporáneo, corresponde ahora retrotraernos en el tiempo histórico para contextualizar ese momento clave de 1869 que nos lleva hasta los inicios de su trayectoria académica universitaria, pero también a la coyuntura determinante de la revolución previa en la que inicia su actividad de compromiso político como parte del grupo de jóvenes krausistas de la generación de 1868.

La integración en el krausismo se produjo tras su llegada a Madrid a finales de la década de 1850 para proseguir los estudios de Derecho que había iniciado en la Universidad de Oviedo y cuya licenciatura obtendrá en julio de 1861. Una incorporación que debió de resultarle tan sencilla como natural, dadas las estrechas relaciones que su padre, Patricio de Azcárate, tenía con las dos figuras claves del primer krausismo español: con Julián Sanz del Río por su coincidencia en varias empresas relacionadas con el cultivo de la filosofía y con su coterráneo Fernando de Castro (la correspondencia entre ambos documenta ya para 1860 la entrada en contacto del joven Gumersindo con el núcleo krausista madrileño) ${ }^{6}$. Pronto entablará relación de amistad con otros discípulos de Sanz del Río, como Nicolás Salmerón o Francisco Giner de los Ríos, al que le unirá una amistad fraternal de por vida. En cualquier caso, en esos años Azcárate parece más inclinado bien al ejercicio profesional de la abogacía, colegiándose en Madrid en 1862, bien al trabajo dentro de la administración, ingresando como auxiliar en la Dirección General del Registro de la Propiedad y del Notariado en diciembre de $1861^{7}$. En este puesto, obtenido por oposición, desarrolló una labor que le llevará hasta ocupar el escalafón de jefe del negociado a comienzos de 1869, así como una protesta ante el Ministerio de Gracia y Justicia que supondrá su cese. Como respuesta va a plantear un contencioso administrativo que a la postre le permitió la reincorporación en

6 Cfr. G. Capellán, Gumersindo de Azcárate. Biografía intelectual, Valladolid, Junta de Castila y León, 2005 , pp. 25 y ss.

7 Gaceta de Madrid, n. ${ }^{\circ} 354,20$ de diciembre de 1861, p. 2. 
marzo de 1871. Un episodio que adquiere su interés porque esa experiencia personal convencerá a Azcárate de que uno de los mayores males de la España del período era la «empleomanía» o el uso político de la administración por parte de los gobiernos para colocar a sus clientelas. La carencia de una administración pública cuyos puestos se consiguieran por oposición y resultaran inamovibles suponía —a su juicio_ - un lastre para la modernización del Estado o para el correcto funcionamiento del régimen constitucional y parlamentario que formalmente proclamaba la teoría política del liberalismo ${ }^{8}$.

También ese desempeño profesional debió de ser fundamental para que cuando se proclame la I Republica en 1873, accediendo su buen amigo Nicolás Salmerón al Ministerio de Gracia y Justicia, se decidiera a colaborar con el gobierno aceptando el puesto de Director General del Registro de la Propiedad y del Notariado. Si bien a los pocos meses dimitirá del cargo que ocupaba en comisión y sin sueldo, lo mismo que hizo el Secretario General del Ministerio de Gracia y Justicia, otro buen amigo krausista, Manuel Ruiz de Quevedo?.

Estos hechos vienen a matizar debidamente una de las ideas generalmente extendidas sobre el primer krausismo español: que su actividad principal fue la especulación filosófica, lo cual les dejó alejados de la práctica, del mundo real. Por el contrario, al menos desde 1860 los principales jóvenes integrantes del krausismo español salieron a la palestra pública no solo para difundir su pensamiento sociopolítico, sino para influir en la opinión pública y poder enmendar el rumbo del país. Revistas como La Razón, Revista Ibérica de Ciencias, Política, Literatura, Artes e Instrucción Pública, La Enseñanza (luego Revista de Instrucción Pública), La Voz del Siglo evidenciaron los sólidos vínculos intelectuales que aunaron a Francisco de Paula Canalejas (Catedrático de Filosofía del Derecho en la Universidad Central), Joaquín Sanromá, Miguel Morayta, Segismundo Moret, Eduardo Pérez Pujol, Gabriel Rodríguez, Félix Bona, García Labiano, Rafael María de Labra, Juan Uña, Juan Facundo Riaño, José Luis y Francisco Giner de los Ríos, Gumersindo de Azcárate...

Instrumentos de difusión de sus ideas a la opinión pública que, a su vez, sirvieron como aglutinante de un grupo que llegará a 1868 defendiendo un ideario que ellos mismos definían como liberalismo radical ${ }^{10}$. O, lo que es lo mismo, el

8 En una de sus obras más influyentes, El régimen parlamentario en la práctica (Madrid, 1885), Azcárate identifica entre los grandes males en el funcionamiento del Estado en España una «tiranía administrativa», fruto de que el Estado asume indebidamente funciones en la adjudicación de expedientes («expedienteo»), la concesión de empleos («empleomanía») y la manipulación de las elecciones («caciquismo»). Y una «tiranía judicial», consistente en el nombramiento por recomendación de los magistrados (cito por la edición de 1933, p. 49).

9 El nombramiento tuvo lugar el 18 de marzo de 1873 y la dimisión el 27 de junio (Gaceta de Madrid, 19 de marzo y 27 de junio de 1873, p.1). Actualmente, el Colegio de Registradores de España concede un premio anual denominado Gumersindo de Azcárate, en reconocimiento a su labor y escritos en ese ámbito del derecho. En relación con ello, vid. C. VATTier Fuenzalida, C., Gumersindo de Azcárate y la renovación de la ciencia del derecho en el siglo XIX, Madrid, Centro de Estudios Registrales, 1998.

10 Un relato detallado de esta acción conjunta y cohesionada del grupo puede verse en G. CAPELLAN, «Liberalismo armónico. La teoría política del primer krausismo español (1860-1868)», Historia y política, n. ${ }^{\circ}$ 
desarrollo de los principios liberales en todas sus manifestaciones y hasta sus últimas consecuencias, tal y como esperaban de la revolución que saludaron con esperanzas de regeneración y progreso en septiembre de 1868. Proclama que en la práctica implicaba la libertad de cultos — que Azcárate entenderá como la coexistencia de una Iglesia y Estado libres en una sociedad libres-, a la vez que como condición ineludible de la libertad de conciencia; la libertad de enseñanza y la libertad industrial y comercial. Unas libertades que debían quedar garantizadas por medio de otras libertades esenciales, las de imprenta, reunión y asociación. Esta última resultaba fundamental para un modelo de organización jurídico-política y social donde el Estado debía ajustar su papel a sus fines jurídicos propios, mientras la sociedad, mediante la libre asociación de los individuos para perseguir sus demás fines vitales, cobraba un protagonismo determinante.

Este punto intermedio entre lo que Azcárate entendía que eran los extremos del liberalismo individualista, que reducía el papel del Estado a la nada, y los del socialismo, que confiaba al Estado la solución de todos los problemas de la sociedad, estaba acorde con la filosofía del derecho krausista. Una filosofía que hizo de la metáfora organicista, trasladada de la naturaleza a la vida social, un medio para comunicar su visión contraria a las teorías atomísticas que concebían la sociedad como un mero agregado de individuos. También para expresar su idea clave de una sociedad autónoma y soberana como centro motor de la vida, para la que usará el término inglés selfgovernment o gobierno del país por el país. Incluso cuando se admitió la necesaria intervención del Estado en problemas tan acuciantes como la denominada cuestión social, Azcárate consideró siempre que debía ser un papel transitorio debido a que la sociedad carecía aún de la madurez para afrontarla por sí misma.

Pero incluso en otros terrenos espinosos, como la acuciante desigualdad económica que había generado la sociedad moderna, consideró que más efectiva que una redistribución de la riqueza por parte del Estado, podía resultar la acción de los individuos para dar un fin social a la riqueza, de acuerdo con una concepción ética que podemos denominar «capitalismo responsable». En el texto con el que nacía a comienzos de 1870 la Asociación Nacional para el Estudio y Reforma de los Presupuestos, que firmaba Azcárate junto a los hermanos Giner de los Ríos, los abogados krausistas Ruiz de Quevedo y Rafael María de Labra o Moret, entre otros, se aseguraba que la asociación es «el gran motor de los progresos de todos los pueblos libres». Porque la asociación es, en realidad, la organización y expresión de la fuerza avasalladora de la opinión pública que propulsa la energía nacional sin que los gobiernos puedan oponerle resistencia, tal y como ejemplifica el

17, Madrid, enero-junio (2007), p. 89-120. «Liberalismo armónico» es una categoría analítica que sirve para caracterizar ese ideario (vid., por ejemplo, la Semblanza incluida en esta misma revista por J. VARELA SUANZES-CARPEGNA, «Un influyente maestro del derecho político español: Vicente Santamaría de Paredes (18531924)», TRC, n. ${ }^{\circ} 34$, pp. 641-658. 
gran ejemplo del pueblo inglés ${ }^{11}$. Convencido de esa necesaria libre iniciativa social en los más diversos temas de la vida, Azcárate se implicará activamente en asociaciones (o sociedades) tan diversas como las constituidas para el progreso de las ciencias, la libertad del arancel, la abolición de la esclavitud, la enseñanza de la mujer...

Para garantizar la seguridad de esas libertades, de los individuos y de su propiedad, se planteaban como imprescindibles la supresión de la pena de muerte, la abolición de la esclavitud y el juicio por jurados. En esta etapa de activista y propagandista del joven Azcárate, la defensa de estos principios se llevó a cabo en esa combinación entre los artículos doctrinales propios del profesor universitario, los escritos de protesta, por ejemplo ante las Cortes, o las conferencias y mítines en foros públicos como el Ateneo, Teatros... Ese fue el caso de las campañas de propaganda para sensibilizar a la opinión pública y presionar al gobierno con el fin de abolir la esclavitud en las colonias españolas. Los krausistas fueron, desde su fundación en 1865, miembros muy activos de la sociedad abolicionista española. Destaca en este terreno el activismo de Rafael María de Labra, que reunirá de nuevo a sus amigos de la generación del 68 en la publicación no académica que quizá mejor expreso el pensamiento político del krausismo durante el Sexenio democrático: El Correo de España. Tanto en esta nueva empresa, como antes en $L a$ Voz o por medio de la traducción de los escritos del krausista alemán K.D.A. Róder que llevó a cabo Francisco Giner de los Ríos, se denunció la vigencia de la pena de muerte, suprimida ya de todas las constituciones de los pueblos civilizados. El mejor argumento en contra de esta idea incompatible con la personalidad humana y los derechos naturales del individuo lo encontraron en la teoría correccional de Röder. Si la función de la pena debía ser la enmienda del individuo para devolverlo a la sociedad en las condicionas previas al delito, la pena de muerte imposibilitaba ese fin primordial. Una visión plenamente acorde con el optimismo antropológico krausista que creía plenamente en la perfectibilidad del ser humano.

La tercera de las garantías para las libertades individuales que Azcárate consideraba que debía consagrar el derecho, en este caso a través del código fundamental, era el juicio por jurados. Si bien este punto será reiterado en sus obras más académicas posteriores o planteado en el Congreso cuando desarrolle su actividad parlamentaria, ya en el Sexenio hizo una temprana defensa de la cuestión desde las páginas de El Correo de España. Desde esta tribuna pretende aportar argumentos teóricos y prácticos para que tanto en la conciencia social como en el orden científico se clarifique y se conceda mayor importancia al tema del jurado, cuya exigencia aflora «siempre que se verifica un movimiento político en sentido liberal» ${ }^{12}$. En ese intento se pone de manifiesto la importancia clave que el

11 Cfr. La Época, n. ${ }^{\circ} 6683$, Madrid, 21 de marzo de 1870, p.1.

12 G. de AzCÁrate, «El Jurado», El Correo de España, n. ${ }^{\circ}$ XVIII, mayo de 1870, pp. 10. 
sistema jurídico-político inglés va a tener para Azcárate. El origen del jurado lo rastrea en las instituciones judiciales del feudalismo, frente a la tradición del juicio por jueces que procede del derecho canónico. En Inglaterra, por haberse conservado muchos elementos de la tradición feudal, ha permanecido el jurado moderno, tomándolo de aquí los Estados Unidos, Una institución que permite una aplicación coherente de la soberanía de todo el pueblo, de la sociedad, para intervenir en todas las esferas del derecho y del poder ${ }^{13}$.

Para Azcárate es un principio indiscutible que por la misma razón que el ciudadano debe participar en el poder legislativo, tanto de forma indirecta como directa por medio del sufragio, o en el poder ejecutivo, merced a la publicidad impuesta a los gobiernos que permiten su control, tiene derecho a tomar parte inmediata en la administración de justicia constituyendo el jurado. Tras analizar en detalle los debates sobre las partes en las que debe intervenir o la capacidad del ciudadano para dirimir estas cuestiones, considera un error la distinción entre el orden criminal y el orden civil del derecho - tal y como hizo la Asamblea Constituyente de 1789, que solamente admitió el jurado para el primero-. A su juicio, la mala aplicación que se ha hecho en este punto en Francia y Alemania proceden de una mala interpretación de Montesquieu (ya señalada por Ahrens) sobre la separación de los poderes en Inglaterra.

En esta coyuntura clave el joven profesor krausista plantea algunas de las ideas centrales de su pensamiento jurídico y político. Solamente me voy a centrar ahora en dos que resultan determinantes, tanto en su acción pública, como en las facetas de escritor y profesor universitario: el Derecho como principio rector de la organización del Estado y —en directa relación con esa concepción-, la accidentalidad de las formas de gobierno.

Derecho fue, no por azar, el nombre elegido para otra empresa publicística emprendida tras la revolución de 1868 junto a su querido Francisco Giner. Por algunos textos posteriores sabemos que para Azcárate la idea del derecho es la que marcaba un avance esencial en la evolución del viejo liberalismo español decimonónico desde la mejor de sus expresiones, la progresista, hacia la que debía ser su seña ineludible de progreso en la sociedad moderna: la democracia. Frente al dogma de la soberanía nacional, defendido por el progresismo como fuente de poder — con lo que Azcárate está de acuerdo—, pero también de derecho, Azcárate niega este último punto estableciendo la primacía del derecho ${ }^{14}$.

13 Para Azcárate el juicio por jurados es condición sine qua non de su ideal del selfgovernment o soberanía de la sociedad, razón por la que siempre defenderá que ésta «debe entender directamente en la declaración y cumplimiento del derecho». Véase el capítulo VII que dedica íntegramente a la institución del jurado en una de sus obras fundamentales, El selfgovernment o la monarquía doctrinaria, Madrid, 1877 (edición del CEPC, 2008, pp. 141-159).

14 G. DE AzCÁRATE, «Olozaga. Origen, ideas y vicisitudes del partido progresista. El Parlamento desde 1840 hasta 1866», en La España del siglo XIX. Colección de Conferencias Históricas. Curso de 1885-86 (El Ateneo), Madrid, Libreria de Don Antonio San Martin, 1886, pp. 5-36. 
Tomando como ejemplo dos casos ya mencionados aquí, esa concepción significaba que, si una mayoría nacional soberana considerara que la esclavitud es positiva, el Estado como institución para el derecho no debería contemplar esa posibilidad porque va contra un derecho esencial, natural, como es el que todo hombre tiene a la libertad. Lo mismo si se planteara establecer la pena de muerte, que va contra el principio esencial de enmienda, además de contra la irrenunciable dignidad humana que define la condición y vida de todo hombre.

En este punto la influencia del krausismo alemán, y concretamente de la filosofía del derecho de $\mathrm{H}$. Ahrens, resultó determinante. Su Curso de derecho natural, que contó con numerosas ediciones en español desde 1841 se convirtió en una obra de referencia en la que se formaron varias generaciones de juristas españoles. Uno de los destacados krausistas de la primera generación, Joaquín María Sanromá, afirmaba que en los años cincuenta del siglo XIX, cuando él era catedrático de Derecho Político en la Universidad de Santiago de Compostela, «Los profesores jóvenes, sin renegar de los viejos, teníamos otro calendario: San Ahrens y compañeros mártires... de la fe krausista». Añadiendo que, de Ahrens, «con ciertas reservas, tomábamos la esencialidad del derecho político: concepto y fin del Estado, noción de la soberanía y del Poder, formas de Gobierno, clasificación de las funciones del Poder, carácter y mecanismos de la representación pública, estructuras constitucionales» ${ }^{15}$.

Y, así fue, en efecto, en el caso de Azcárate, que siguió a Ahrens en un punto esencial del derecho político, las formas de Estado. Llevando la cuestión más allá de los debates clásicos de la teoría política sobre las formas de gobierno, y tomando explícitamente en esto como referencia las ideas de Kant, Ahrens reconoció como forma del Estado el Rechstaat o Estado de derecho. De este modo, el derecho quedaba establecido «como principio ético y objetivo, al cual debe someterse la voluntad de todos, y como principio orgánico que garantiza a todos los miembros y a todas las partes su posición y su acción libre y la participación en el ejercicio de todos los poderes públicos» ${ }^{16}$. Y en ese sentido se define un modelo de sociedad y de organización estatal que permite hablar de «el reinado del derecho». Este principio se opone justamente al despotismo, la otra forma de Estado reconocida por Kant, que ahora quedaba definido como «la voluntad arbitraria personal que se pone en lugar del derecho y de la ley libremente consentida». Esta primacía de la idea del derecho estuvo en el corazón mismo del liberalismo democrático que propagaban no sólo los jóvenes krausistas de la generación de 1868, sino otros autores destacados del momento — y en buena medida afines - como Emilio Castelar.

Por la misma vía llegó Azcárate a otra idea central de su pensamiento jurídico-político: la accidentalidad de las formas de gobierno. Uno de los lemas que

15 J.M. Sanromá, Mis memorias, Madrid, Hijos de M. G. Hernández, 1894, tomo II, p. 288.

$16 \mathrm{H}$. Ahrens, Curso de derecho natural o de filosofía del derecho, Madrid, Carlos Bailly-Bailliere, 1873 (3. edición española). 
figuraba en la cabecera de la revista que puso en marcha tras la revolución de 1868 junto con Giner de los Ríos, Derecho, rezaba así: «indiferencia de las formas de gobierno». Nótese la relevancia de este principio en un contexto histórico español en el que la monarquía aparecía de forma mayoritaria identificada con el propio Estado. Una concepción que precisamente entró en pleno conflicto con las culturas políticas de corte republicano en pleno ascenso en la coyuntura de 1868, tensión que no hizo sino agravarse durante el Sexenio democrático hasta la proclamación de la I República en 1873.

Para afrontar en la práctica esa situación Azcárate acudió pertrechado del marco teórico provisto por la filosofía del derecho de Ahrens, pero que tenía también antecedentes tan destacados como el eminente jurista y político francés Odilón Barrot. Para estos autores «el Estado no tiene una forma política», sino «también una forma de cultura». Es decir, que las formas políticas no son nunca independientes «de un cúmulo de circunstancias históricas, del genio de un pueblo, y del estado de su cultura». La conclusión que deriva de semejante planteamiento es que ninguna de las formas políticas particulares posee un valor absoluto. $\mathrm{O}$, lo que es lo mismo, que las formas de gobierno clásicas, entre ellas la monarquía o la república no deben ser rechazadas o aceptadas en abstracto, sino que deben analizarse en la práctica, en un contexto histórico, social y cultural concreto. Así, Ahrens creía que la Monarquía, «puede ofrecer por su principio la más eficaz garantía para el desarrollo pacífico y continuo de todos los intereses sociales» ${ }^{17}$. Lo único que esta institución debe hacer si quiere conservarse en la Europa moderna es cumplir con «la practica sincera y equitativa del sistema representativo».

El propio Azcárate, en su habitual estilo de contrastar la teoría jurídica con la práctica histórica acudiendo a la comparación entre países, mencionaba el ejemplo de su admirada Inglaterra que muestra «la historia de una Monarquía bajo la cual el pueblo ha adquirido toda la libertad de una República». También se refiere a Bélgica, cuya constitución de 1831 siempre le pareció ejemplar, por la conformación efectiva de una monarquía parlamentaria que «elige para jefe uno que fue modelo de reyes constitucionales y consigue ver los altos poderes del Estado tan admirablemente organizados en sus esferas independientes, que se vivifican y alimentan periódicamente en la fecunda fuente de la soberanía popular». A la nómina se sumaba también Italia, país que de la mano de Víctor Manuel, «rey electo, querido y amado de los italianos», había logrado «abrir las puertas del poder, no ya al liberalismo, sino a la misma democracia» ${ }^{18}$.

17 H. Ahrens, Curso de derecho natural, op. cit., p. 569. Para la formulación de Odilón Barrot, remito a su obra traducida y difundida en la España del Sexenio, De la centralización y sus efectos, Madrid, 1869 (existe una edición facsimilar moderna, Madrid, Civitas, 1997 con prólogo de E. García Enterría).

18 Cfr. G. DE Azcarate, El régimen parlamentario en la práctica, op. cit., p.147. Para el caso inglés véase el capítulo dedicado a la obra de Erskine May, Democracia en Europa que incluye en sus Tratados de política. Resúmenes y juicios críticos. Madrid, Imprenta de Enrique de la Riva, 1883 (cap. 1; la cita en p. 19). 
Sobre esa base teórica se explica que en la coyuntura histórica de 1869 Azcárate apoyara la posibilidad de una monarquía democrática, que finalmente se concretó en la figura de Amadeo de Saboya. Pero también se entiende, sin que supusiera incurrir en ninguna incoherencia o contradicción doctrinal, que posteriormente desde su militancia progresista-democrática participara en diversos partidos republicanos, pero sin dejar de hacer gala de un accidentalismo no siempre bien aceptado en la época. Esa postura le permitiría aceptar la monarquía de Alfonso XIII, siempre que bajo ese aspecto formal reinaran las libertades, el derecho y la democracia.

Unos principios que Azcárate consideró plasmados en el espíritu y la letra de la constitución de 1869, si bien no se le escapaba que a partir de ese marco jurídico esencial la clave radicaba después en su aplicación práctica, en «el cumplimiento de lo dispuesto en la Constitución del Estado» ${ }^{19}$. Esta primera etapa del joven Azcárate, inmerso en la coyuntura histórica, político-social y cultural marcada por el Sexenio democrático, resulta determinante, ya que durante todo el período posterior la Constitución de 1869 se conservará no sólo como símbolo de un orden liberal democrático de referencia (y a recuperar), sino como el contrapunto al orden jurídico establecido en la Constitución de 1876, marco bajo el cual se desarrolló el resto de su vida.

\section{LA RESTAURACIÓN: EL SELFGOVERMENT Y EL MODELO CONSTITUCIONAL DE INGLATERRA}

Como buen profesor de legislación comparada, Azcárate no sólo izó la bandera de la constitución de 1869 en sus múltiples escritos, discursos o intervenciones políticas en la larga carrera parlamentaria iniciada en 1886, sino que desde el mismo momento en el que se produjo la Restauración de Alfonso XII a finales de 1874 y el nuevo régimen tomó carta de naturaleza en el código fundamental aprobado en junio de 1876, miró hacia otras realidades constitucionales, como Inglaterra, en busca de alternativas a la deficiente aplicación del gobierno parlamentario en España. Este fue denostado bajo el calificativo de «Monarquía doctrinaria», en alusión a la influencia de los doctrinaires franceses que traslucía el conservadurismo canovista, principal artífice del edificio político sobre el que se erigió la Restauración.

De hecho, la restauración monárquica marcará el devenir inmediato, tanto personal como académico, de Azcárate y sus principales amigos krausistas. Desde el Ministerio de Fomento del primer gobierno de la Restauración, con el Marqués de Orovio al frente, se llevaron a cabo una serie de medidas destinadas a restablecer el control de la enseñanza universitaria, que bajo el principio de la libertad se había transformado notablemente durante los años del Sexenio.

$19 \ll$ El jurado», art. cit., p.10. 
Todo comenzó en febrero de 1875 con un Real Decreto del Ministerio de Fomento que derogaba ciertos artículos del Decreto de 21 de octubre de 1868 «relativos a textos y programas», al tiempo que llevaba a cabo «el restablecimiento en esta parte de la legislación que venía rigiendo desde $1845 »^{20}$. Detrás de este Decreto y la circular subsiguiente se hallaba el deseo del gobierno conservador de controlar las enseñanzas impartidas desde las Cátedras, con la prohibición expresa de que se expusieran ideas contrarias a la monarquía y a la religión católica, dos pilares esenciales del nuevo régimen. Una circunstancia que tenía precedentes en los años 60, en las denominadas polémicas contra los «textos vivos», en expresa referencia a la influencia ejercida por los jóvenes profesores krausistas y demócratas desde sus cátedras universitarias ${ }^{21}$. Un episodió que concluyó en sendas cuestiones universitarias, cuando los gobiernos ultraconservadores de la recta final de la monarquía isabelina expulsaron de sus cátedras a Castelar, Sanz del Río, Salmerón o Francisco Giner de los Ríos en 1865 y 1868. En el trasfondo subyacía también un doble debate. De un lado, el relativo a la libertad de la ciencia que sustentaba la libertad de cátedra. Este fue el primer argumento esgrimido por Azcárate, Giner y Salmerón en 1875 para negarse a cumplir las ordenes ministeriales, protagonizando el primer gran episodio de defensa de la libertad de cátedra de la universidad española contemporánea. De otro lado, el referente a la legislación vigente, pero que, en realidad, revelaba un debate central sobre las últimas constituciones proclamadas en España, así como sus evidentes diferencias. Azcárate y sus amigos argumentaron que la legislación y la constitución vigente (a esas alturas aún la de 1869) les concedía la mencionada libertad de cátedra. Mientras que el Ministro Orovio evidenciaba la mentalidad del nuevo gobierno al retrotraerse a la constitución moderada de 1845. A su juicio, como expresara sin ningún ambage en el Congreso de los diputados pocos meses más tarde, desde los sucesos de 1873 — es decir, la I República_- la constitución de 1869 había quedado «decapitada, asesinada y enterrada».

La cuestión universitaria acabó con la protesta y dimisión de numerosos profesores, así como con la separación de sus cátedras y exilio de Salmerón, Giner y Azcárate. Dado que no serán repuestos en sus cátedras hasta el primer gobierno liberal de 1881, los profesores krausistas pusieron en marcha una institución educativa desde la que proseguir su labor docente, La Institución Libre de Enseñanza (ILE), que se convertirá durante los seis decenios de su existencia en un referente pedagógico, científico e intelectual. Azcárate fue socio fundador, profesor de derecho y rector de la ILE.

Fue en ese largo lustro fuera de la cátedra oficial cuando Azcárate escribió dos de sus principales textos centrados en la organización jurídico-política del Estado:

20 Los principales documentos implicados en: Cuestión universitaria. Documentos coleccionados por M. Ruiz de Quevedo referentes a los profesores separados, dimisionarios y suspensos, Madrid, A. J. Alaria, 1876.

21 Puede verse mi introducción a E. Soler, Derecho político, Athenaica, Sevilla, 2015. 
El selfgovernment y la monarquía doctrinaria (1877) y La constitución inglesa y la politica en el continente (1878). También entonces inició su obra jurídica de mayor envergadura, Ensayo sobre la historia del derecho de propiedad y su estado actual en Europa (1879-1883, en 3 vols.). En este sentido, y a diferencia de otros eminentes juristas de la época, Azcárate no publicó ningún texto o manual de éxito fruto de su labor docente en el ámbito del derecho. Sobre la legislación comparada, que tantos años enseñó en el doctorado de derecho de la Universidad Central, publicó tempranamente un breve plan general, pero no contamos con más texto que los apuntes que sus estudiantes tomaban y las contestaciones al programa que «publicaban» manuscritas para disgusto de Don Gumersindo ${ }^{22}$.

La primera de esas obras fue en realidad una recopilación de varios artículos escritos tras su separación de la cátedra en 1875 y publicados de forma sucesiva en Revista de España a partir de enero de 1876. En una reseña del libro aparecida en Revista Europea, el escritor e intelectual Armando Palacio Valdés consideraba El self-government y la monarquía doctrinaria como un trabajo que «aborda con resolución los problemas del derecho político moderno». En ella — proseguía su comentario- el autor discurre en la mayor parte de sus capítulos «con tal madurez y precisión de juicio, que bastan para colocarlo entre los primeros pensadores, no sólo españoles sino extranjeros ${ }^{23}$. En la obra abordaba, en efecto, temas relevantes de los que entonces solía ocuparse la disciplina del derecho político y otros candentes en la propia política del momento: la legalidad de los partidos políticos, cercenada durante la primera etapa de la Restauración; la legitimidad de las revoluciones; que Azcárate, a pesar de su preferencia por el reformismo como vía de cambio gradual y pacífico, admitía cuando la sociedad era privada del ejercicio de sus libertades y derechos; los gobiernos personales, como el cesarismo y la dictadura; el parlamentarismo, capítulo en el que avanza la falsedad de su práctica en España y las consecuencias negativas de su sistemático falseamiento desde el mismo proceso electoral; el jurado, donde vuelve a defender su necesidad, al tiempo que denuncia su inexistencia bajo la monarquía doctrinaria; la centralización, en la que contrapone sus consecuencias perjudiciales, como en el caso

22 Programa de legislación comparada. Curso de 1897-1898 (Madrid, 1897). La primera parte recoge la asignatura del programa estructurada en 57 lecciones con Alemania, Francia, Inglaterra y Estados Unidos, además de España, como países de referencia para la comparación de la organización jurídica. La segunda parte, bajo el título «Legislación comparada por G. Azcárate. Contestaciones adaptadas al programa de dicha asignatura. Cuaderno 1. ${ }^{\circ}$ (Madrid, Montserrat 10, Litografía, 1897) expone el desarrollo de cada una de las lecciones, tomando como referencia esencial el manual del profesor estadounidense de la Universidad de Columbia F. Johson Goodnow, Derecho administrativo comparado: Análisis de los sistemas administrativos de los Estados Unidos, Inglaterra, Francia y Alemania (aparecido en 1894 y traducido luego al español por La España Moderna, 1897-1898, 2 vols.). El núcleo de la asignatura es el análisis de las teorías sobre la división de los poderes del Estado, que toma como punto de partida a Montesquieu, corrigiendo muchos de sus postulados.

23 Cfr. Revista Europea, tomo IX, n. ${ }^{\circ}$ 153, 28 de enero de 1878, pp. 115-116. Si bien Valdés es más conocido como escritor y crítico literario, atesoraba un buen conocimiento del ámbito jurídico, ya que se había licenciado en Leyes e incluso ocupó temporalmente la Cátedra de Derecho civil en la Universidad de Oviedo en sustitución de F. Aramburu. 
francés y español, frente a los efectos beneficiosos de la descentralización administrativa, como evidenciaban los ejemplos inglés y norteamericano; o las constituciones irreformables.

Me detendré brevemente en este último punto (capítulo IV de la citada obra) porque resultaba del máximo interés en el contexto en el que acababa de aprobarse la constitución de 1876 (el artículo se publica en julio de ese mismo año). Curiosamente este artículo desató una enorme y duradera polémica por una afirmación secundaria dentro de su contenido estrictamente jurídico: que España había sufrido un gran atraso cultural y científico durante los últimos tres siglos debido a que el Estado no había amparado la libertad de la ciencia. Juicio que enervó a Menéndez Pelayo, generando toda una serie de artículos que acabarían engrosando la denominada «Polémica sobre la ciencia española».

Pero lo realmente relevante del artículo es, en primer lugar, su concepto de constitución que se ramifica en tres líneas. La constitución natural, que se refiere a la sociedad general, a todos los aspectos de su vida. La constitución jurídica, que se refiere solamente a la realización del derecho y establece las reglas que presiden la vida en esa esfera. Y la constitución política, subordinada a la anterior y que «se refiere tan solo a la organización y funciones del poder». De semejante concepción se derivan dos matizaciones esenciales. De un lado, la necesaria distinción entre la sociedad (el todo) y el Estado (la parte, la sociedad jurídica) que tantos tratadistas confunden dando lugar a un error capital. De otro, la aspiración en las corrientes científicas dominantes a desligar el orden político respecto al orden jurídico, «como si el derecho político no fuera una rama del derecho» ${ }^{24}$.

En ese marco debe entenderse una de las reivindicaciones centrales de los primeros liberal-demócratas españoles de que, tal y como lo expresaron cuando se debatía la constitución de 1869, los derechos naturales de los individuos son no sólo inalienables, sino también imprescriptibles y, en consecuencia, no deben — no pueden - formar parte del texto constitucional (lo cual implicaría que los otorga la propia constitución). Para Azcárate este dualismo apreciable en las constituciones modernas procede de reunir dos elementos esencialmente distintos: la declaración de los derechos del hombre, naturales o individuales - lo que los ingleses denominan garantías o libertades- que forman parte del derecho de la personalidad o sustantivo; y la organización del poder, las reglas en las que se asienta la organización del Estado, asunto del derecho público o adjetivo. En puridad cuando se emplea el nombre constitución política se refiere solamente a esta última parte, mientras resulta más apropiado usar el de Código fundamental cuando se consignan principios jurídicos esenciales que desenvuelven luego los códigos y las leyes ${ }^{25}$.

24 G. DE AzCÁRATE, El selfgovernment... (ed. 2008), pp.70-71

25 Ibíd., pp. 72-73. 
Fijado de esa forma el concepto de constitución, Azcárate lleva a cabo en su texto una crítica frontal a algunos presupuestos claves de la teoría política dominante, como el contractualismo, la co-soberanía rey-pueblo o la idea de que la voluntad es fuente de soberanía. Al hacerlo refutaba que la constitución fuera el resultado de un pacto entre dos instancias soberanas (rey-Cortes), así como la creencia de que recoge la esencia de una sociedad y que, por lo tanto, es inmutable. Para Azcárate, imbuido de una filosofía de la historia krausista en la que la ley natural del progreso rige la marcha de la humanidad, la organización de una sociedad se haya sometida a evolución. Es decir, cambia con los tiempos, teniendo esas transformaciones que reflejarse tanto en las leyes ordinarias como en las fundamentales.

Con este planteamiento, el ilustre jurista español refutaba la monarquía doctrinaria vigente en España que — a su juicio — concibe el poder y la constitución como un pacto entre el monarca y los individuos, sacralizando el orden constitucional como el de la propia figura del rey y legitimando ese orden sin aceptar cambios. Pero, como la soberanía pertenece solamente a la sociedad, que se gobierna por sí misma, para sí misma (selfgovernment), ella siempre conserva la capacidad para dotarse de nuevas leyes, para reformar cualquier institución o realidad cuando el sentimiento general de la nación, expresado en la opinión pública, está dispuesto a ello. De acuerdo con esa concepción dinámica de la historia, en la que la soberanía radica en la sociedad, parecía lógico que la constitución debía ajustarse a las necesidades y al sentir de la sociedad en cada momento mediante su reforma. En la práctica política Azcárate se integrará en la minoría republicana en el Congreso a partir de 1886, primero, y fundará el partido Reformista (1912), más tarde, poniendo siempre como condición sine qua non para aceptar la monarquía liberal restaurada que se reformara el artículo 11 de la constitución de 1876: la tolerancia religiosa debía sustituirse por la libertad de cultos (que implicaba la irrenunciable libertad de conciencia de los individuos) $)^{26}$.

Esta crítica abierta al nuevo régimen jurídico-político de la Restauración, así como el planteamiento de una propuesta alternativa articulada en torno al principio del selfgovernment tuvo, de alguna manera, su continuidad en otra de las obras mencionadas: La constitución inglesa y la política en el continente.

El origen de esta monografía fueron los debates celebrados en el Ateneo de Madrid en 1878 en torno a dos preguntas: «¿Debe la Gran Bretaña a la Constitución política el carácter a la vez progresivo y pacífico de su actual civilización?» $\mathrm{Y}$, en caso afirmativo, que fue la creencia dominante entre todos los oradores

26 Sobre esta condición del reformismo republicano para aceptar el régimen de la Restauración siempre que fuera capaz de democratizarse, véase M. SuÁrez CORTINA, Los caballeros de la razón. Cultura institucionista y democracia parlamentaria en la España liberal (Genueve ediciones, 2018) p. 90 y ss. En ese mismo contexto Azcárate publicó una obra clave para la entender la candente cuestión religiosa, Minuta de un testamento anotada por W... (Madrid, V. Suárez, 1876). Hay una edición moderna a cargo de Elías Díaz (Ediciones de cultura Popular, 1967) y otra más reciente de la Fundación Giner de los Ríos (2017). 
participantes en la discusión, surgía la segunda interrogación: "¿qué hay en ella de propio y particular de aquel país, y qué de común que pueda aplicarse a los demás pueblos?»

Azcárate inicia su texto reproduciendo un juicio que consideraba exacto, que «Inglaterra es el modelo, la fuente de todos los experimentos constitucionales que se han hecho en los tiempos modernos». Afirmación contundente a la que sigue la consideración de que, por lo general, los pueblos europeos se habían contentado con «copiar de segunda mano, acudiendo a Francia, donde es fácil leer la letra, pero no el espíritu, que con frecuencia falta, en vez de apelar a Inglaterra donde el espíritu es todo, la letra nada» ${ }^{27}$.

Una cita que nos remite directamente a dos aspectos a tener en cuenta. En primer lugar, la necesidad de indagar en los principios que impregnaban una constitución caracterizada por no tener un registro escrito al uso. En segundo lugar, que pese a esa circunstancia el sistema constitucional de Inglaterra resultaba bien conocido y reconocido a esas alturas del siglo XIX. En ese sentido debe recordarse el papel pionero fundamental que desempeñó la exposición del jurista suizo De Lolme, cuya exitosa obra sobre la constitución de Inglaterra fue publicada inicialmente en francés (Amsterdam, 1771) y más tarde en inglés (Londres, 1785). Merced a su exposición, que buscaba precisamente «un acercamiento constitucional» de Francia y el resto de Europa a Inglaterra, la constitución inglesa toma cuerpo para convertirse en modelo de organización jurídica del principio de libertad ${ }^{28}$.

La traducción de la obra al español, publicada en singulares coyunturas de la historia constitucional española del siglo XIX, como 1812 y 1847, contribuyó a difundir esa visión positiva — y hasta paradigmática— de la constitución inglesa. Tarea a la que también contribuyó la obra de Walter Bagehot, The English Constitution, que Adolfo Posada verterá al español al comenzar el siglo $\mathrm{XX}^{29}$. En ese contexto histórico se enmarca la mirada que hacia el constitucionalismo inglés se lanza desde España al iniciarse la Restauración. Azcárate se muestra convencido del provecho que del estudio de esa constitución se puede sacar porque no sólo es el ejemplo de la materialización práctica del principio de libertad que ha informado la política moderna, sino de la democracia hacia la que irremisiblemente marcha la humanidad (convicción que Tocqueville había contribuido a extender), pero a la que se resistía España —en su opinión-.

Entre los aspectos de la constitución inglesa susceptibles de adoptar - $-\mathrm{y}$ adaptar - en otros pueblos modernos Azcárate destaca una vez más el principio

27 «Prólogo» a La constitución inglesa... (1878) p.VII.

28 Sigo las consideraciones de Bartolomé Clavero en su estudio preliminar a: De Lolme, J.L. Constitución de Inglaterra, Madrid, Centro de Estudios Políticos y Constitucionales, 1992, p. 32. Clavero habla de una auténtica «invención constitucional» por parte de De Lolme al tomar el «derecho inglés» y convertirlo en «derecho constitucional» (pp. 28-32).

29 Al respecto, vid. la edición y estudio de Joaquín Varela Suanzes-Carpegna a: W. BAGEHOT, $L a$ Constitución inglesa, Madrid, CEPC, 2010. 
del selfgovernment que la define. Tanto en su acepción antigua de autonomía local, que permite una sana descentralización administrativa, como en el moderno de la soberanía de la sociedad, que en Inglaterra se cumple de manera ejemplar. Allí la sociedad lo es todo y la opinión pública es la fuerza social sin cuyo respaldo ninguno de los poderes del Estado puede ejercer su acción. Junto a todo ello resalta una separación de poderes en la que destaca la independencia del judicial, la fortaleza del legislativo, que hace efectivo el régimen parlamentario, y el papel de la monarquía, ya que el jefe del Estado no ejerce las prerrogativas regias, de acuerdo con la fórmula de que el rey reina, pero no gobierna.

A modo de colofón, debe señalarse que en el transcurso de su actividad parlamentaria Azcárate puso al servicio de la política de distintos gobiernos sus conocimientos jurídicos, colaborando en la elaboración de diversas leyes aprobadas durante los lustros finales del siglo XIX y los primeros del xx. Fue el caso de la reforma de la legislación electoral que llevó al famoso artículo 29 para la proclamación automática de candidatos en los distritos, con el fin de evitar la lucha política y las prácticas fraudulentas asociadas al caciquismo imperante en la época que tanto le repugnaban. La colaboración con el gobierno de Antonio Maura para elaborar el proyecto de Ley de bases para la reforma de la administración local ${ }^{30}$. O la célebre ley para la represión de la usura, conocida como ley Azcárate, promulgada en 1908 y vigente — con algunas modificaciones— hasta la actualidad.

El prestigio y respecto que en la vida pública española se había ganado el maestro al iniciarse el siglo xx hicieron que, primero el gobierno liberal de Canalejas (Instituto de Trabajo, 1902) y después el gobierno conservador de Francisco Silvela (Instituto de Reformas Sociales, 1903), contaran con Azcárate para abordar una de las grandes preocupaciones del período: la cuestión social. Con el fin de estudiar las condiciones de la creciente clase obrera, así como de buscar fórmulas para mejorar su condición, se llevaron a cabo desde 1883 una serie de iniciativas gubernamentales que culminaron con el Instituto de Reformas Sociales presidido por Gumersindo de Azcárate desde su creación hasta que, en pleno desarrollo de una de sus sesiones, el maestro acabó sus días en diciembre de 1917.

Desde esa posición se afanó en aplicar otro de los grades ideales krausistas, la metáfora de la armonía. La armonía social, preferible a la lucha de clases, debía solucionar los conflictos entre el capital y el trabajo. Para ello Azcárate, revestido de su autoridad, su prestigio, pero también de su talante abierto, pleno de tolerancia y neutralidad, medió entre patrones y obreros, para proponer acciones y legislación que ayudaran a facilitar lo que denominaba «el advenimiento del cuarto estado a la vida». Al fin y al cabo, la democracia hacia la que los pueblos

30 Su permanente interés en la descentralización administrativa y el fortalecimiento del municipalismo puede verse en la recopilación de sus escritos sobre la materia: G. DE AzCARATE, Municipalismo y regionalismo, Madrid, INAP, 1979. 
modernos debían orientar su devenir no era otra cosa que la plena incorporación del elemento popular en todas las esferas de la sociedad.

La labor de Azcárate en este ámbito culminaría una trayectoria vital que dejó una profunda huella en sus contemporáneos. No es de extrañar que uno de los jóvenes intelectuales más crítico con la vieja política que representaba la Restauración escribiera al hilo del fallecimiento del maestro que «Se nos va con Azcárate el último ejemplar de una casta de hombres que creía en las cosas superiores», el último de «los hombres de 1869», la persona en la que esa generación adquirió «la más pura y sencilla calidad» ${ }^{31}$.

Pero quizá el testimonio que mejor podría cerrar esta Semblanza es el que aporta un eminente jurista con quien compartió la labor desde la cátedra y múltiples iniciativas institucionales, Adolfo Posada: «Azcárate fue para mí —y para tantos-, pero estimo que para mí de modo excepcional, consejero y guía. Lo que yo le debo a Azcárate no sabría decirlo y menos explicarlo, ¿para qué? Le debo en buena parte la formación de mi íntimo ser moral» ${ }^{32}$.

$$
\text { *** } *
$$

TitLE: Gumersindo de Azcárate: Law, Self-government and the Constitution of England

AвSTRACT: Gumersindo de Azcárate (León, 1840_ Madrid, 1917) was one the most influential Law professor and politician in Contemporary Spain. He was an active member of the so-call krausist movement that played a major role in Spanish culture and politics after the revolution of 1868. From both, journals and University he defended the liberal and democratic principles that lead to his ideal: a rule of law. In that context he supported the new constitution of 1869, regarded by Azcarate for the rest of his life as the best one in Spanish modern history. When the Restoration took place in 1874 and a constitutional Monarchy inspired in French doctinaires' political theory was set up, Azcárate criticised it proposing to look over the Constitution of England as a model. According to his interpretation of English constitutional system, the principle of self-government or the sovereignty of society was the key principle for building a true democratic parliamentary government based on the free association of individuals, a decentralized administration, an independent judicial power and public opinion as the very source, guide and limit of all the powers of the State. From 1886 up to 1916 Azcárate became Member of the Parliament as representative of the republican party and was actively involve in the Intitute for Social Reforms (1903) that tried to improve the condition of the working classes.

Resumen: Gumersindo de Azcárate (León, 1840-Madrid, 1917) fue uno de los más influyentes catedráticos de derecho y políticos de la España contemporánea. Fue un miembro activo del denominado movimiento krausista que desempeñó un importante papel en la cultura y política española tras la revolución de 1868. Desde diferentes revistas y desde la propia Universidad defendió los principios liberales y democráticos que conducian al establecimiento de su ideal: Estado de derecho. En ese contexto apoyó la nueva constitución de 1869, que Azcárate considerará siempre un referente y el mejor código fundamental de la España moderna. Con la Restauración en 1874 de la Monarquía inspirada en el doctrinarismo francés Azcárate se mostró muy crítico y propuso dirigir la mirada hacia la constitución de Inglaterra como el modelo jurídico-político a tener en cuenta. A su juicio el sistema constitucional inglés se articulaba en torno al principio del self-goverment o

31 J. Ortega y Gasset, «Don Gumersindo de Azcárate ha muerto anoche. Su vida y sus obras», El Sol, 15-12-1917.

32 Posada, A., Fragmentos de mis memorias, Oviedo, Universidad de Oviedo, 1983, p. 321. 
soberanía de la sociedad a partir del cual se construía un régimen parlamentario democrático con una administración descentralizada, un poder judicial independiente y una opinión pública que actuaba a la vez como fuente, guía y límite de los distintos poderes del Estado. Entre 1886 y 1916 Azcárate fue Diputado en el Congreso de los Diputados por el partido republicano y se implicó activamente, como presidente del Instituto de Reformas Sociales (1903), en la mejora de las condiciones de vida las clases obreras.

KEY WORDS: Azcárate, Rule of Law, Selfgovernment, Constitution of England, Spanish Constitution of 1869 .

Palabras Clave: Azcárate, Estado de Derecho, Selfgovernment, Constitución de Inglaterra, Constitución española de 1869.

FECHA DE RECEPCIÓN: 11.06.2019

FECHA DE ACEPTACIÓN: 29.07.2019 